\title{
An Extended Auxiliary Function Method and Its Application in mKdV Equation
}

\author{
Yafeng Xiao, ${ }^{1}$ Haili Xue, ${ }^{2}$ and Hongqing $\mathrm{Zhang}^{3}$ \\ ${ }^{1}$ Department of Mathematics, North University of China, Taiyuan 030051, China \\ ${ }^{2}$ Software School, North University of China, Taiyuan 030051, China \\ ${ }^{3}$ School of Mathematical Sciences, Dalian University of Technology, Dalian 116024, China \\ Correspondence should be addressed to Yafeng Xiao; yafengxiao@126.com
}

Received 29 November 2012; Accepted 22 February 2013

Academic Editor: Farzad Khani

Copyright (c) 2013 Yafeng Xiao et al. This is an open access article distributed under the Creative Commons Attribution License, which permits unrestricted use, distribution, and reproduction in any medium, provided the original work is properly cited.

\begin{abstract}
An extended auxiliary function method is presented for constructing exact traveling wave solutions to nonlinear partial differential equations. The main idea of this method is to take full advantage of the solutions to the elliptic equation to construct exact traveling wave solutions for nonlinear partial differential equations. $\mathrm{mKdV}$ equation is chosen to illustrate the application of the extended auxiliary function method. Consequently, more new exact traveling wave solutions are derived that are not obtained by the previously known methods.
\end{abstract}

\section{Introduction}

The investigation of exact solutions, in particular solitons, for nonlinear mathematical physics equations is an important and interesting subject. Many authors have paid attention to this subject. And many effective methods have been presented, such as Bäcklund transformation, Darboux transformation, variable separation approach, various tanh methods, Painlevé method, generalized hyperbolic-function method, homogeneous balance method, and similarity reduction method [1-17].

With the rapid development of computer algebraic systems like Maple or Mathematical, many powerful algebraic methods have been presented to obtain a great many exact solutions (especially traveling wave solutions) for nonlinear partial differential equation (NLPDE), for example, sinecosine method [18], Fu's method [19], the hyperbolic tangent function expansion method $[8,20]$, Riccati equation expansion method $[9,21]$, the generalized Riccati equation method [22], auxiliary ordinary differential equation method [23], modified extended tanh method [24], Fan's unified algebraic method [25], the extended Fan's subequation method [26], and so on [26-36]. Generally speaking, these methods have a common characteristic: the possible solutions are constructed from the known functions or the solutions for some simple and solvable nonlinear ordinary differential equations such as sine-cosine function, tanh function, Jacobi elliptic function or the Riccati equation, the coupled Riccati equation, and sinh-Gordon equation. Recently, Xu presents the elliptic equation expansion method to construct new and more general solutions of NLPDE [37]. The main purpose of this paper is to summarize and extend Xu's method to unified basic frame so that it can be used to obtain more exact traveling wave solutions for NLPDE. For illustration, we apply the extended method to the modified Korteweg-de Vries (mKdV) equation and get successfully many new exact solutions.

This paper is organized as follows. In Section 2, we give the main steps of our extended algorithm for computing exact solutions of NLPDE. In Section 3, many families of traveling wave solutions of $\mathrm{mKdV}$ equation are obtained. Finally, some conclusions and discussions are provided in Section 4.

\section{The Extended Elliptic Equation Method and Its Algorithm}

In the following we would like to outline the main steps of our method. 
Step 1 (reduce the nonlinear PDE to the nonlinear ODE). For a Given nonlinear partial differential equation with the physical field $u(x, t)$ in two variables $x, t$

$$
\Delta\left(u, u_{t}, u_{x}, u_{t t}, u_{x t}, u_{x x}, \ldots\right)=0
$$

We consider its traveling wave solution in the form

$$
u(x, t)=U(\xi), \quad \xi=k(x-c t),
$$

where $k$ and $c$ are constants to be determined later. Substituting (2) into (1) gives rise to a nonlinear ordinary differential equation with constant coefficients

$$
\Theta\left(U, U^{\prime}, U^{\prime \prime}, \ldots\right)=0 .
$$

Step 2 (set the series of formal solutions). To seek the traveling wave solutions to (3), in [37] Xu assumes that (3) has the solutions in the form of

$$
U(\xi)=a_{0}+\sum_{i=1}^{n} a_{i} \varphi^{i}(\xi),
$$

with the new variable $\varphi(\xi)$ satisfying the elliptic equation

$$
\varphi^{\prime 2}=A \varphi(\varphi-\alpha)(\varphi-\beta)(\varphi-\gamma),
$$

where $A, \alpha, \beta$, and $\gamma$ are constants and $n$ is the integer to be determined later.

In order to get more exact solutions for (3), here we assume that (3) has the following formal solutions:

$$
\begin{aligned}
U(\xi)=a_{0}+\sum_{i=1}^{n}[ & a_{i} \varphi^{i}(\xi)+b_{i} \varphi^{i-1}(\xi) \varphi^{\prime}(\xi) \\
& \left.+c_{i} \varphi^{-i}(\xi)+d_{i} \varphi^{-i}(\xi) \varphi^{\prime}(\xi)\right] .
\end{aligned}
$$

Step 3 (determine the truncation expansion terms in (6)). In order to obtain the value of $n$ in (6). We define the degree of $U(\xi)$ as $D[U(\xi)]=n$ which gives rise to the degrees of other expressions as

$$
D\left[\frac{d^{q} U}{d \xi^{q}}\right]=n+q, \quad D\left[U^{p}\left(\frac{d^{q} u}{d \xi^{q}}\right)^{s}\right]=n p+s(n+q) .
$$

Therefore we can get the value of $n$ in (6) by balancing the highest-order contributions from such terms in (1) or (3). If $n$ is a nonnegative integer, we first make the transformation $U=w^{n}$.

Step 4 (lead to the set of algebraic equations). We substitute (6) along with (5) into (3) and then set to zero the coefficients of like powers of $\varphi^{i} \varphi^{\prime j}(i=0,1,2, \ldots ; j=0,1)$ to obtain a set of nonlinear algebraic equations with respect to the unknowns $a_{0}, a_{i}, b_{i}, c_{i}$, and $d_{i}(i=1,2, \ldots)$.

Step 5 (solve the algebraic equations). By solving the overdetermined system of nonlinear algebraic equations by use of symbolic computation system Maple, we can get these unknowns $a_{0}, a_{i}, b_{i}, c_{i}$ and $d_{i}(i=1,2, \ldots)$.
Step 6 (solve (5)). Motivated by [37], we obtain some special solutions for (5) which are now listed in the following.

Case 1. If $A>0$ and $\alpha<0<\beta<\gamma$, (5) has the solution

$$
\begin{gathered}
\varphi(\xi)=\frac{\alpha \beta s n^{2}\left(\alpha_{1} \xi\right)}{\alpha-\beta c n^{2}\left(\alpha_{1} \xi\right)}, \quad \alpha_{1}=\frac{1}{2} \sqrt{A \gamma(\beta-\alpha)}, \\
m=\sqrt{\frac{\beta(\gamma-\alpha)}{\gamma(\beta-\alpha)}} .
\end{gathered}
$$

Case 2. If $A>0$ and $\alpha<\beta<0<\gamma$, (5) has the solution

$$
\begin{gathered}
\varphi(\xi)=\frac{\alpha \gamma s n^{2}\left(\alpha_{1} \xi\right)}{\gamma-\alpha c n^{2}\left(\alpha_{1} \xi\right)}, \quad \alpha_{1}=\frac{1}{2} \sqrt{A \beta(\alpha-\gamma)}, \\
m=\sqrt{\frac{\alpha(\gamma-\beta)}{\beta(\gamma-\alpha)}} .
\end{gathered}
$$

Case 3. If $A<0$ and $0<\alpha<\beta<\gamma$, (5) has the solution

$$
\begin{gathered}
\varphi(\xi)=\frac{\alpha(\gamma-\beta) s n^{2}\left(\alpha_{1} \xi\right)-\beta(\gamma-\alpha)}{(\gamma-\beta) s n^{2}\left(\alpha_{1} \xi\right)-(\gamma-\alpha)}, \\
\alpha_{1}=\frac{1}{2} \sqrt{A \beta(\alpha-\gamma)}, \\
m=\sqrt{\frac{\alpha(\gamma-\beta)}{\beta(\gamma-\alpha)}} .
\end{gathered}
$$

Case 4. If $A<0$ and $\alpha<0<\beta<\gamma$, (5) has the solution

$$
\begin{gathered}
\varphi(\xi)=\frac{\beta \gamma}{\gamma+(\beta-\gamma) s n^{2}\left(\alpha_{1} \xi\right)}, \\
\alpha_{1}=\frac{1}{2} \sqrt{-A \gamma(\beta-\alpha)}, \\
m=\sqrt{\frac{\alpha(\beta-\gamma)}{\gamma(\beta-\alpha)}} .
\end{gathered}
$$

Case 5. If $A<0$ and $\alpha<\beta<0<\gamma$, (5) has the solution

$$
\begin{gathered}
\varphi(\xi)=\frac{\beta \gamma s n^{2}\left(\alpha_{1} \xi\right)}{\beta-\gamma c n^{2}\left(\alpha_{1} \xi\right)}, \\
\alpha_{1}=\frac{1}{2} \sqrt{A \alpha(\gamma-\beta)}, \\
m=\sqrt{\frac{\gamma(\alpha-\beta)}{\alpha(\gamma-\beta)}} .
\end{gathered}
$$

Step 7 (obtain the exact solutions to (3)). By inserting each solution to the above set of algebraic equations into (6) and making use of the solutions (8)-(12), some new types of elliptic function solutions for (3) can be obtained.

Remark 1. The method proposed here is more general than Xu's method for finding exact solutions for NLPDE. When $b_{i}=0, c_{i}=0$ and $d_{i}=0(i=1,2, \ldots)$, our method can degenerate to Xu's method. 


\section{The Application of the Elliptic Equation Expansion Method}

In the following, we will apply the extended elliptic equation method to construct new explicit solutions of $\mathrm{mKdV}$ equation [1]:

$$
u_{t}+\alpha u^{2} u_{x}+\beta u_{x x x}=0
$$

where $\alpha$ and $\beta$ are constants.

The $\mathrm{mKdV}$ equation is of important significance in many branches of nonlinear science field. When $\alpha=6$, (13) is called the positive $\mathrm{mKdV}$ equation, while $\alpha=-6$, (13) is called the negative $\mathrm{mKdV}$ equation. The $\mathrm{mKdV}$ equation appears in many fields such as acoustic waves in certain anharmonic lattices, Alfvén waves in a collisionless plasma, transmission lines in Schottky barrier, models of traffic congestion, ion acoustic solitons, elastic media, and so forth. It possesses many remarkable properties such as miura transformation, conservation laws, inverse scattering transformation, bilinear transformation, N-solitons, breather solutions, Bäcklund transformation, Painlevé integrability, Darboux transformation, and doublyperiodic solutions.

According to Step 1 in Section 2, in order to look for the traveling wave solutions for (13), we make the following traveling wave transformation:

$$
u(x, t)=U(\xi), \quad \xi=k(x-c t),
$$

and substitute (14) into (13). Then (13) changes into the form

$$
-c U^{\prime}+\alpha U^{2} U^{\prime}+\beta k^{2} U^{\prime \prime \prime}=0 .
$$

According to Steps 2 and 3 in Section 2, by balancing the highest-order partial derivative term $U^{\prime \prime \prime}$ with the highest nonlinear term $U^{2} U^{\prime}$ in (15), we can obtain $n=1$. So we have

$$
U=a_{0}+a_{1} \varphi+b_{1} \varphi^{\prime}+\frac{c_{1}}{\varphi}+d_{1} \frac{\varphi^{\prime}}{\varphi}
$$

where $a_{0}, a_{1}, b_{1}, c_{1}$ and $d_{1}$ are constants to be determined later and $\varphi$ satisfies (5).

According to Step 4 in Section 2, with the aid of Maple, substituting (16) into (15) yields a set of algebraic equations for $\varphi^{i} \varphi^{\prime j}(i=-1,0,1 ; j=0,1)$. Setting the coefficients of these terms $\varphi^{i} \varphi^{\prime j}$ of the resulting system's numerator to zero yields a set of overdetermined algebraic equations with respect to the unknowns $a_{0}, a_{1}, b_{1}, c_{1}$, and $d_{1}$ :

$$
\begin{gathered}
\frac{\alpha}{3 \beta k^{2}} b_{1}^{3} A=0, \quad \frac{\alpha}{\beta k^{2}} c_{1}^{2} d_{1}=0, \\
\frac{\alpha}{\beta k^{2}} a_{1} b_{1}^{2} A=0, \quad \frac{\alpha}{3 \beta k^{2}} c_{1}^{3}=0 \\
-\frac{\alpha}{3 \beta k^{2}}\left(-3 b_{1}^{2} A d_{1}+b_{1}^{3} A \beta+b_{1}^{3} A \alpha+b_{1}^{3} A \gamma\right)=0, \\
6 b_{1} A-\frac{\alpha}{3 \beta k^{2}}\left(-3 a_{1}^{2} b_{1}-b_{1}^{3} A \alpha \gamma-b_{1}^{3} A \alpha \beta\right. \\
+3 b_{1}^{2} A d_{1} \beta+3 b_{1}^{2} A d_{1} \alpha-b_{1}^{3} A \beta \gamma \\
\left.-3 b_{1} A d_{1}^{2}+3 b_{1}^{2} A d_{1} \gamma\right)=0,
\end{gathered}
$$

$2 d_{1} A-3 h_{3} a_{1}^{2} d_{1}-3 h_{3} b_{1}^{2} A d_{1} \alpha \gamma-3 h_{3} b_{1}^{2} A d_{1} \alpha \beta$

$$
-3 h_{3} b_{1}^{2} A d_{1} \beta \gamma-3 b_{1} A \gamma-3 b_{1} A \beta
$$$$
+h_{3} b_{1}^{3} A \alpha \beta \gamma+3 h_{3} b_{1} A d_{1}^{2} \gamma-3 b_{1} A \alpha
$$$$
+3 h_{3} b_{1} A d_{1}^{2} \alpha-h_{3} d_{1}^{3} A
$$$$
-6 h_{3} a_{0} a_{1} b_{1}+3 h_{3} b_{1} A d_{1}^{2} \beta=0,
$$

$-\frac{\alpha}{\beta k^{2}}\left(-a_{0} b_{1}^{2} A-2 a_{1} b_{1} A d_{1}+a_{1} b_{1}^{2} A \alpha\right.$

$$
\left.+a_{1} b_{1}^{2} A \gamma+a_{1} b_{1}^{2} A \beta\right)=0,
$$

$$
\begin{gathered}
2 a_{1} A-\frac{\alpha}{3 \beta k^{2}}\left(6 a_{1} b_{1} A d_{1} \beta-3 a_{1} b_{1}^{2} A \beta \gamma-3 a_{1} b_{1}^{2} A \alpha \gamma\right. \\
-3 a_{1} b_{1}^{2} A \alpha \beta-3 b_{1}^{2} A c_{1}+3 a_{0} b_{1}^{2} A \alpha \\
+6 a_{1} b_{1} A d_{1} \alpha-a_{1}^{3}-3 a_{1} d_{1}^{2} A+3 a_{0} b_{1}^{2} A \gamma \\
\left.-6 a_{0} b_{1} A d_{1}+6 a_{1} b_{1} A d_{1} \gamma+3 a_{0} b_{1}^{2} A \beta\right)=0 \\
-\frac{3}{2} a_{1} A \alpha-\frac{3}{2} a_{1} A \beta-\frac{3}{2} a_{1} A \gamma \\
-\frac{\alpha}{\beta k^{2}}\left(2 a_{0} b_{1} A d_{1} \alpha-2 a_{1} b_{1} A d_{1} \alpha \beta-2 a_{1} b_{1} A d_{1} \alpha \gamma\right. \\
-a_{0} b_{1}^{2} A \alpha \beta-2 b_{1} A c_{1} d_{1}+b_{1}^{2} A c_{1} \alpha \\
+2 a_{0} b_{1} A d_{1} \beta-a_{0} a_{1}^{2}+b_{1}^{2} A c_{1} \beta \\
-a_{0} b_{1}^{2} A \alpha \gamma-a_{0} b_{1}^{2} A \beta \gamma+2 a_{0} b_{1} A d_{1}-a_{0} d_{1}^{2} A \\
+b_{1}^{2} A c_{1} \gamma+a_{1} d_{1}^{2} A \beta+a_{1} b_{1}^{2} A \alpha \beta \gamma \\
\left.+a_{1} d_{1}^{2} A \gamma-2 a_{1} b_{1} A d_{1} \beta \gamma+a_{1} d_{1}^{2} A \alpha\right)=0 \\
a_{1} A(\alpha \beta+\beta \gamma+\alpha \gamma)-\frac{c}{\beta k^{2}} a_{1} \\
\alpha k^{2}\left(2 b_{1} A c_{1} d_{1} \gamma+2 b_{1} A c_{1} d_{1} \beta-a_{0}^{2} a_{1}+2 b_{1} A c_{1} d_{1} \alpha\right.
\end{gathered}
$$




$$
\begin{aligned}
& -a_{1} d_{1}^{2} A \beta \gamma-a_{1} d_{1}^{2} A \alpha \gamma-a_{1} d_{1}^{2} A \alpha \beta \\
& -b_{1}^{2} A c_{1} \beta \gamma-a_{1}^{2} c_{1}-b_{1}^{2} A c_{1} \alpha \gamma \\
& -b_{1}^{2} A c_{1} \alpha \beta+a_{0} d_{1}^{2} A \gamma+a_{0} d_{1}^{2} A \beta-c_{1} d_{1}^{2} A \\
& +a_{0} d_{1}^{2} A \alpha+2 a_{1} b_{1} A d_{1} \alpha \beta \gamma+a_{0} b_{1}^{2} A \alpha \beta \gamma \\
& \left.-2 a_{0} b_{1} A d_{1} \alpha \gamma-2 a_{0} b_{1} A d_{1} \beta \gamma-2 a_{0} b_{1} A d_{1} \alpha \beta\right)=0 .
\end{aligned}
$$

According to Step 5 in Section 2, by use of the Maple software package "Charsets" by Dongming Wang, which is based on the Wu-elimination method [38], solving the previous overdetermined algebraic equations, we can get the following three sets of explicit expressions for $a_{0}, a_{1}, b_{1}, c_{1}$, and $d_{1}$.

Family 1. Consider

$$
\begin{gathered}
a_{0}=\mp \frac{1}{4} \sqrt{-\frac{6 \beta k^{2} A}{\alpha}}(\alpha+\beta+\gamma), \\
a_{1}= \pm \sqrt{-\frac{6 \beta k^{2} A}{\alpha}} \\
b_{1}=0, \quad c_{1}=0, \quad d_{1}=0, \\
\frac{c}{\beta k^{2}}=-\frac{A}{8}\left(3 \beta^{2}-2 \alpha \beta-2 \beta \gamma+3 \alpha^{2}-2 \alpha \gamma+3 \gamma^{2}\right) .
\end{gathered}
$$

Family 2. Consider

$$
\begin{aligned}
& a_{0}=0, \quad a_{1}=0, \\
& b_{1}=0, \quad c_{1}=0, \\
& d_{1}= \pm \sqrt{-\frac{6 \beta k^{2}}{\alpha}}
\end{aligned}
$$

Family 3. Consider

$$
\begin{gathered}
a_{0}=0, \quad a_{1}= \pm \sqrt{-\frac{3 \beta k^{2} A}{2 \alpha}} \\
b_{1}=0, \quad c_{1}=0, \quad d_{1}= \pm \sqrt{-\frac{3 \beta k^{2}}{2 \alpha}}, \\
\frac{c}{\beta k^{2}}=-\frac{1}{2} A(\alpha \beta+\beta \gamma+\alpha \gamma) .
\end{gathered}
$$

According to Step 7 in Section 2, substituting (18)-(20) into (16), respectively, and combining Step 6 in Section 2, we get the following traveling wave solutions to (15).
Family 1. We obtained the following exact solutions:

$$
\begin{aligned}
& u_{11}=\mp \frac{1}{4} \sqrt{-\frac{6 \beta k^{2} A}{\alpha}}(\alpha+\beta+\gamma) \\
& \pm \sqrt{-\frac{6 \beta k^{2} A}{\alpha}} \frac{\alpha \beta s n^{2}\left(\alpha_{1} \xi\right)}{\alpha-\beta c n^{2}\left(\alpha_{1} \xi\right)} \\
& m=\sqrt{\frac{\beta(\gamma-\alpha)}{\gamma(\beta-\alpha)}}, \\
& \alpha_{1}=\frac{1}{2} \sqrt{A \gamma(\beta-\alpha)} \text {, } \\
& \frac{c}{\beta k^{2}}=-\frac{A}{8}\left(3 \beta^{2}-2 \alpha \beta-2 \beta \gamma+3 \alpha^{2}-2 \alpha \gamma+3 \gamma^{2}\right), \\
& u_{12}= \pm \sqrt{-\frac{6 \beta k^{2}}{\alpha}} \frac{2 \alpha_{1}(\alpha-\beta) c n\left(\alpha_{1} \xi\right) d n\left(\alpha_{1} \xi\right)}{s n\left(\alpha_{1} \xi\right)\left[\alpha-\beta c n^{2}\left(\alpha_{1} \xi\right)\right]} \\
& \alpha_{1}=\frac{1}{2} \sqrt{A \gamma(\beta-\alpha)}, \quad m=\sqrt{\frac{\beta(\gamma-\alpha)}{\gamma(\beta-\alpha)}}, \\
& u_{13}= \pm \sqrt{-\frac{3 \beta k^{2} A}{2 \alpha}} \frac{\alpha \beta s n^{2}\left(\alpha_{1} \xi\right)}{\alpha-\beta c n^{2}\left(\alpha_{1} \xi\right)} \\
& \pm \sqrt{-\frac{3 \beta k^{2}}{2 \alpha}} \frac{\alpha_{1}(\alpha-\beta) c n\left(\alpha_{1} \xi\right) d n\left(\alpha_{1} \xi\right)}{s n\left(\alpha_{1} \xi\right)\left(\alpha-\beta c n^{2}\left(\alpha_{1} \xi\right)\right)}, \\
& \alpha_{1}=\frac{1}{2} \sqrt{A \gamma(\beta-\alpha)}, \quad m=\sqrt{\frac{\beta(\gamma-\alpha)}{\gamma(\beta-\alpha)}}, \\
& \frac{c}{\beta k^{2}}=-\frac{1}{2} A(\alpha \beta+\beta \gamma+\alpha \gamma),
\end{aligned}
$$

where $\xi=k(x-c t), A>0$, and $\alpha<0<\beta<\gamma$.

Family 2. We obtained the following exact solutions:

$$
\begin{gathered}
u_{21}=\mp \frac{1}{4} \sqrt{-\frac{6 \beta k^{2} A}{\alpha}}(\alpha+\beta+\gamma) \\
\pm \sqrt{-\frac{6 \beta k^{2} A}{\alpha}} \frac{\alpha \gamma s n^{2}\left(\alpha_{1} \xi\right)}{\gamma-\alpha c n^{2}\left(\alpha_{1} \xi\right)}, \\
m=\sqrt{\frac{\alpha(\gamma-\beta)}{\beta(\gamma-\alpha)}} \\
\alpha_{1}=\frac{1}{2} \sqrt{A \beta(\alpha-\gamma)},
\end{gathered}
$$




$$
\begin{aligned}
& \frac{c}{\beta k^{2}}=-\frac{A}{8}\left(3 \beta^{2}-2 \alpha \beta-2 \beta \gamma+3 \alpha^{2}-2 \alpha \gamma+3 \gamma^{2}\right), \\
& u_{22}= \pm \sqrt{-\frac{6 \beta k^{2}}{\alpha}} \frac{2 \alpha_{1}(\gamma-\alpha) c n\left(\alpha_{1} \xi\right) d n\left(\alpha_{1} \xi\right)}{s n\left(\alpha_{1} \xi\right)\left[\gamma-\alpha c n^{2}\left(\alpha_{1} \xi\right)\right]} \\
& \alpha_{1}=\frac{1}{2} \sqrt{A \beta(\alpha-\gamma)}, \quad m=\sqrt{\frac{\alpha(\gamma-\beta)}{\beta(\gamma-\alpha)}}, \\
& u_{23}= \pm \sqrt{-\frac{3 \beta k^{2} A}{2 \alpha}} \frac{\alpha \gamma s n^{2}\left(\alpha_{1} \xi\right)}{\gamma-\alpha c n^{2}\left(\alpha_{1} \xi\right)} \\
& \pm \sqrt{-\frac{3 \beta k^{2}}{2 \alpha}} \frac{2 \alpha_{1}(\gamma-\alpha) c n\left(\alpha_{1} \xi\right) d n\left(\alpha_{1} \xi\right)}{s n\left(\alpha_{1} \xi\right)\left(\gamma-\alpha c n^{2}\left(\alpha_{1} \xi\right)\right)} \\
& \alpha_{1}=\frac{1}{2} \sqrt{A \beta(\alpha-\gamma)}, \quad m=\sqrt{\frac{\alpha(\gamma-\beta)}{\beta(\gamma-\alpha)}}, \\
& \frac{c}{\beta k^{2}}=-\frac{1}{2} A(\alpha \beta+\beta \gamma+\alpha \gamma)
\end{aligned}
$$

where $\xi=k(x-c t), A>0$, and $\alpha<\beta<0<\gamma$.

Family 3 . We obtained the following exact solutions:

$$
\begin{aligned}
u_{31}= & \mp \frac{1}{4} \sqrt{-\frac{6 \beta k^{2} A}{\alpha}}(\alpha+\beta+\gamma) \\
& \pm \sqrt{-\frac{6 \beta k^{2} A}{\alpha} \frac{\alpha(\gamma-\beta) s n^{2}\left(\alpha_{1} \xi\right)-\beta(\gamma-\alpha)}{(\gamma-\beta) s n^{2}\left(\alpha_{1} \xi\right)-(\gamma-\alpha)},} \\
\alpha_{1} & =\frac{1}{2} \sqrt{A \beta(\alpha-\gamma)}, \quad m=\sqrt{\frac{\alpha(\gamma-\beta)}{\beta(\gamma-\alpha)}}, \\
\frac{c}{\beta k^{2}}= & -\frac{A}{8}\left(3 \beta^{2}-2 \alpha \beta-2 \beta \gamma+3 \alpha^{2}-2 \alpha \gamma+3 \gamma^{2}\right),
\end{aligned}
$$

$u_{32}$

$$
\begin{gathered}
= \pm \sqrt{-\frac{6 \beta k^{2}}{\alpha}} \\
\times \frac{2 \alpha_{1}(\alpha-\beta)(\beta-\gamma)(\gamma-\alpha) s n\left(\alpha_{1} \xi\right) c n\left(\alpha_{1} \xi\right) d n\left(\alpha_{1} \xi\right)}{\left[\alpha(\gamma-\beta) s n^{2}\left(\alpha_{1} \xi\right)-\beta(\gamma-\alpha)\right]\left[(\gamma-\beta) s n^{2}\left(\alpha_{1} \xi\right)-(\gamma-\alpha)\right]} \\
\alpha_{1}=\frac{1}{2} \sqrt{A \beta(\alpha-\gamma)}, \quad m=\sqrt{\frac{\alpha(\gamma-\beta)}{\beta(\gamma-\alpha)}},
\end{gathered}
$$

$$
\begin{aligned}
& u_{33} \\
& = \pm \sqrt{-\frac{3 \beta k^{2}}{2 \alpha}} \\
& \times \frac{2 \alpha_{1}(\alpha-\beta)(\beta-\gamma)(\gamma-\alpha) s n\left(\alpha_{1} \xi\right) c n\left(\alpha_{1} \xi\right) d n\left(\alpha_{1} \xi\right)}{\left[\alpha(\gamma-\beta) s n^{2}\left(\alpha_{1} \xi\right)-\beta(\gamma-\alpha)\right]\left[(\gamma-\beta) s n^{2}\left(\alpha_{1} \xi\right)-(\gamma-\alpha)\right]} \\
& \pm \sqrt{-\frac{3 \beta k^{2} A}{2 \alpha}} \frac{\alpha(\gamma-\beta) s n^{2}\left(\alpha_{1} \xi\right)-\beta(\gamma-\alpha)}{(\gamma-\beta) s n^{2}\left(\alpha_{1} \xi\right)-(\gamma-\alpha)}, \\
& \frac{c}{\beta k^{2}}=-\frac{1}{2} A(\alpha \beta+\beta \gamma+\alpha \gamma), \quad \alpha_{1}=\frac{1}{2} \sqrt{A \beta(\alpha-\gamma)}, \\
& m=\sqrt{\frac{\alpha(\gamma-\beta)}{\beta(\gamma-\alpha)}},
\end{aligned}
$$

where $\xi=k(x-c t), A<0$ and $0<\alpha<\beta<\gamma$.

Family 4 . We obtained the following exact solutions:

$$
\begin{aligned}
& u_{41}=\mp \frac{1}{4} \sqrt{-\frac{6 \beta k^{2} A}{\alpha}}(\alpha+\beta+\gamma) \\
& \pm \sqrt{-\frac{6 \beta k^{2} A}{\alpha}} \frac{\beta \gamma}{\gamma+(\beta-\gamma) s n^{2}\left(\alpha_{1} \xi\right)}, \\
& m=\sqrt{\frac{\alpha(\beta-\gamma)}{\gamma(\beta-\alpha)}}, \\
& \alpha_{1}=\frac{1}{2} \sqrt{-A \gamma(\beta-\alpha)} \text {, } \\
& \frac{c}{\beta k^{2}}=-\frac{A}{8}\left(3 \beta^{2}-2 \alpha \beta-2 \beta \gamma+3 \alpha^{2}-2 \alpha \gamma+3 \gamma^{2}\right) \text {, } \\
& u_{42}= \pm \sqrt{-\frac{6 \beta k^{2}}{\alpha}} \frac{2 \alpha_{1}(\gamma-\beta) \operatorname{sn}\left(\alpha_{1} \xi\right) c n\left(\alpha_{1} \xi\right) d n\left(\alpha_{1} \xi\right)}{\gamma+(\beta-\gamma) s n^{2}\left(\alpha_{1} \xi\right)}, \\
& \alpha_{1}=\frac{1}{2} \sqrt{-A \gamma(\beta-\alpha)}, \quad m=\sqrt{\frac{\alpha(\beta-\gamma)}{\gamma(\beta-\alpha)}}, \\
& u_{43}= \pm \sqrt{-\frac{3 \beta k^{2} A}{2 \alpha}} \frac{\beta \gamma}{\gamma+(\beta-\gamma) s n^{2}\left(\alpha_{1} \xi, m\right)} \\
& \pm \sqrt{-\frac{3 \beta k^{2}}{2 \alpha}} \frac{2 \alpha_{1}(\gamma-\beta) s n\left(\alpha_{1} \xi\right) c n\left(\alpha_{1} \xi\right) d n\left(\alpha_{1} \xi\right)}{\gamma+(\beta-\gamma) s n^{2}\left(\alpha_{1} \xi\right)}, \\
& \alpha_{1}=\frac{1}{2} \sqrt{-A \gamma(\beta-\alpha)}, \quad m=\sqrt{\frac{\alpha(\beta-\gamma)}{\gamma(\beta-\alpha)}}, \\
& \frac{c}{\beta k^{2}}=-\frac{1}{2} A(\alpha \beta+\beta \gamma+\alpha \gamma) \text {, }
\end{aligned}
$$

where $\xi=k(x-c t), A<0$, and $\alpha<0<\beta<\gamma$. 
Family 5. We obtained the following exact solutions:

$$
\begin{aligned}
& u_{51}=\mp \frac{1}{4} \sqrt{-\frac{6 \beta k^{2} A}{\alpha}}(\alpha+\beta+\gamma) \\
& \pm \sqrt{-\frac{6 \beta k^{2} A}{\alpha}} \frac{\beta \gamma s n^{2}\left(\alpha_{1} \xi\right)}{\beta-\gamma c n^{2}\left(\alpha_{1} \xi\right)}, \\
& m=\sqrt{\frac{\gamma(\alpha-\beta)}{\alpha(\gamma-\beta)}}, \\
& \alpha_{1}=\frac{1}{2} \sqrt{A \alpha(\gamma-\beta)} \text {, } \\
& \frac{c}{\beta k^{2}}=-\frac{A}{8}\left(3 \beta^{2}-2 \alpha \beta-2 \beta \gamma+3 \alpha^{2}-2 \alpha \gamma+3 \gamma^{2}\right) \text {, } \\
& u_{52}= \pm \sqrt{-\frac{6 \beta k^{2}}{\alpha}} \frac{2 \alpha_{1}(\beta-\gamma) c n\left(\alpha_{1} \xi\right) d n\left(\alpha_{1} \xi\right)}{s n\left(\alpha_{1} \xi\right)\left[\beta-\gamma c n^{2}\left(\alpha_{1} \xi\right)\right]}, \\
& \alpha_{1}=\frac{1}{2} \sqrt{A \alpha(\gamma-\beta)}, \quad m=\sqrt{\frac{\gamma(\alpha-\beta)}{\alpha(\gamma-\beta)}}, \\
& u_{53}= \pm \sqrt{-\frac{3 \beta k^{2} A}{2 \alpha}} \frac{\beta \gamma s n^{2}\left(\alpha_{1} \xi\right)}{\beta-\gamma c n^{2}\left(\alpha_{1} \xi\right)} \\
& \pm \sqrt{-\frac{3 \beta k^{2}}{2 \alpha}} \frac{2 \alpha_{1}(\beta-\gamma) c n\left(\alpha_{1} \xi\right) d n\left(\alpha_{1} \xi\right)}{\operatorname{sn}\left(\alpha_{1} \xi\right)\left[\beta-\gamma c n^{2}\left(\alpha_{1} \xi\right)\right]}, \\
& \alpha_{1}=\frac{1}{2} \sqrt{A \alpha(\gamma-\beta)}, \quad m=\sqrt{\frac{\gamma(\alpha-\beta)}{\alpha(\gamma-\beta)}}, \\
& \frac{c}{\beta k^{2}}=-\frac{1}{2} A(\alpha \beta+\beta \gamma+\alpha \gamma) \text {, }
\end{aligned}
$$

where $\xi=k(x-c t), A<0$, and $\alpha<\beta<0<\gamma$.

Remark 2. The previous solutions obtained here, to our knowledge, are all new families of rational formal solutions to $\mathrm{mKdV}$ equation.

\section{Conclusion and Discussions}

In summary, we have presented the extended elliptic equation method and its algorithm. As a result, some new exact traveling wave solutions for the $\mathrm{mKdV}$ equation are obtained which may be useful for describing certain nonlinear physical phenomena. The method which we have proposed in this paper is a standard, direct, and computerized method, which allow us to do complicated and tedious algebraic calculation. It is shown that the algorithm can be also applied to other NLPDE in mathematical physics. In addition, a natural problem is that whether the extended elliptic equation method can be further extended to generate more types of solutions of NLPDE in nonlinear science. Hence, further study of the extended elliptic equation method is needed.

\section{Conflict of Interests}

The authors declare that they have no conflict of interests.

\section{Acknowledgments}

Many thanks are due to suggestions from the referees and support from the National Natural Science Foundation of China (Grant no. 61171179) and the Young Scientists Fund of the National Natural Science Foundation of China (Grant no. 10901145).

\section{References}

[1] M. J. Ablowitz and P. A. Clarkson, Solitons, Nonlinear Evolution Equations and Inverse Scattering, Cambridge University Press, New York, NY, USA, 1991.

[2] V. B. Matveev and M. A. Salle, Darboux Transformations and Solitons, Springer, Berlin, Germany, 1991.

[3] Y. Chen, Z. Y. Yan, and H. Q. Zhang, "Exact solutions for a family of variable-coefficient "Reaction-Duffing" equations via the Backlund transformation," Theoretical and Mathematical Physics, vol. 132, no. 1, pp. 970-975, 2002.

[4] B. Li, Y. Chen, and H. Q. Zhang, "Auto-backlund transformations and exact solutions for the generalized two-dimensional kortewegdde vries-burgers-type equations and burgers-type equations," Zeitschrift fr Naturforschung A, vol. 58, no. 7-8, p. 464, 2003.

[5] R. Hirota and J. Satsuma, "Soliton solutions of a coupled Korteweg-de Vries equation," Physics Letters A, vol. 85, no. 8-9, pp. 407-408, 1981.

[6] S.-Y. Lou, "On the coherent structures of the Nizhnik-NovikovVeselov equation," Physics Letters A, vol. 277, no. 2, pp. 94-100, 2000.

[7] E. J. Parkes and B. R. Duffy, "Travelling solitary wave solutions to a compound KdV-Burgers equation," Physics Letters A, vol. 229, no. 4, pp. 217-220, 1997.

[8] W. Hereman, "Exact solitary wave solutions of coupled nonlinear evolution equations using MACSYMA," Computer Physics Communications, vol. 65, no. 1-3, pp. 143-150, 1991.

[9] E. G. Fan, "Extended tanh-function method and its applications to nonlinear equations," Physics Letters A, vol. 277, no. 4-5, pp. 212-218, 2000.

[10] Z. Y. Yan, "The new extended Jacobian elliptic function expansion algorithm and its applications in nonlinear mathematical physics equations," Computer Physics Communications, vol. 153, no. 2, pp. 145-154, 2003.

[11] Z. Y. Yan and H. Q. Zhang, "New explicit solitary wave solutions and periodic wave solutions for Whitham-Broer-Kaup equation in shallow water," Physics Letters A, vol. 285, no. 5-6, pp. 355$362,2001$.

[12] Y. Chen, X. D. Zheng, B. Li, and H. Q. Zhang, "New exact solutions for some nonlinear differential equations using symbolic computation," Applied Mathematics and Computation, vol. 149, no. 1, pp. 277-298, 2004.

[13] Q. Wang, Y. Chen, B. Li, and H. Q. Zhang, "New exact travelling wave solutions for the shallow long wave approximate equations," Applied Mathematics and Computation, vol. 160, no. 1, pp. 77-88, 2005.

[14] B. Li, Y. Chen, and H. Q. Zhang, "Exact travelling wave solutions for a generalized Zakharov-Kuznetsov equation," Applied 
Mathematics and Computation, vol. 146, no. 2-3, pp. 653-666, 2003.

[15] R. Conte and M. Musette, "Link between solitary waves and projective Riccati equations," Journal of Physics A, vol. 25, no. 21, pp. 5609-5623, 1992.

[16] C. A. Gómez, A. H. Salas, and B. Acevedo Frias, "Exact solutions to KdV6 equation by using a new approach of the projective Riccati equation method," Mathematical Problems in Engineering, vol. 2010, Article ID 797084, 10 pages, 2010.

[17] Z. Y. Yan, H. Q. Zhang, and E. G. Fan, "New explicit travelling wave solutions for a class of nonlinear evolution equations," Acta Physica Sinica, vol. 48, no. 1, pp. 1-5, 1999.

[18] C. A. Gómez and A. H. Salas, "Exact solutions for the generalized BBM equation with variable coefficients," Mathematical Problems in Engineering, vol. 2010, Article ID 498249, 10 pages, 2010.

[19] Z. T. Fu, S. Liu, and S. Liu, "New transformations and new approach to find exact solutions to nonlinear equations," Physics Letters A, vol. 299, no. 5-6, pp. 507-512, 2002.

[20] Z. B. Li and Y. P. Liu, "RATH: a Maple package for finding travelling solitary wave solutions to nonlinear evolution equations," Computer Physics Communications, vol. 148, no. 2, pp. 256-266, 2002.

[21] Z. Y. Yan, "New explicit travelling wave solutions for two new integrable coupled nonlinear evolution equations," Physics Letters A, vol. 292, no. 1-2, pp. 100-106, 2001.

[22] F. D. Xie, Y. Zhang, and Z. S. Lü, "Symbolic computation in nonlinear evolution equation: application to $(3+1)$-dimensional Kadomtsev-Petviashvili equation," Chaos, Solitons and Fractals, vol. 24, no. 1, pp. 257-263, 2005.

[23] Sirendaoreji and S. Jiong, "Auxiliary equation method for solving nonlinear partial differential equations," Physics Letters A, vol. 309, no. 5-6, pp. 387-396, 2003.

[24] E. Yomba, "Construction of new soliton-like solutions for the $(2+1)$ dimensional KdV equation with variable coefficients," Chaos, Solitons and Fractals, vol. 21, no. 1, pp. 75-79, 2004.

[25] E. G. Fan and Y. C. Hon, "A series of travelling wave solutions for two variant Boussinesq equations in shallow water waves," Chaos, Solitons \& Fractals, vol. 15, no. 3, pp. 559-566, 2003.

[26] E. Yomba, “The extended Fan's sub-equation method and its application to $\mathrm{KdV}-\mathrm{MKdV}$, BKK and variant Boussinesq equations," Physics Letters A, vol. 336, no. 6, pp. 463-476, 2005.

[27] H. Triki, A. Yildirim, T. Hayat, O. M. Aldossar, and A. Biswas, "Topological and non-topological soliton solutions of the Bretherton equation," Proceedings of the Romanian Academy A, vol. 13, no. 2, pp. 103-108, 2012.

[28] G. Ebadi, A. H. Kara, M. D. Petkovic, A. Yildirim, and A. Biswas, "Solitons and conserved quantities of the Ito equation," Proceedings of the Romanian Academy A, vol. 13, no. 3, pp. 215224, 2012.

[29] H. Triki, A. Yıldırım, T. Hayat, O. M. Aldossary, and A. Biswas, "Shock wave solution of Benney-Luke equation," Romanian Journal of Physics, vol. 57, no. 7-8, pp. 1029-1034, 2012.

[30] G. Ebadi, N. Yousefzadeh, H. Triki, A. Yildirim, and A. Biswas, "Envelope solitons, periodic waves and other solutions to Boussinesq-Burgers equation," Romanian Reports in Physics, vol. 64, no. 4, pp. 915-932, 2012.

[31] M. Antonova and A. Biswas, "Adiabatic parameter dynamics of perturbed solitary waves," Communications in Nonlinear Science and Numerical Simulation, vol. 14, no. 3, pp. 734-748, 2009.
[32] L. Girgis and A. Biswas, "Soliton perturbation theory for nonlinear wave equations," Applied Mathematics and Computation, vol. 216, no. 7, pp. 2226-2231, 2010.

[33] E. V. Krishnan, H. Triki, M. Labidi, and A. Biswas, "A study of shallow water waves with Gardner's equation," Nonlinear Dynamics, vol. 66, no. 4, pp. 497-507, 2011.

[34] M. Labidi, H. Triki, E. V. Krishnan, and A. Biswas, "Soliton solutions of the long-short wave equation with power law nonlinearity," Journal of Applied Nonlinear Dynamics, vol. 1, no. 2, pp. 125-140, 2012.

[35] E. V. Krishnan, S. Kumar, and A. Biswas, "Solitons and other nonlinear waves of the Boussinesq equation," Nonlinear Dynamics, vol. 70, no. 2, pp. 1213-1221, 2012.

[36] A. Biswas and M. S. Ismail, "1-soliton solution of the coupled KdV equation and Gear-Grimshaw model," Applied Mathematics and Computation, vol. 216, no. 12, pp. 3662-3670, 2010.

[37] G. Q. Xu, "An elliptic equation method and its applications in nonlinear evolution equations," Chaos, Solitons and Fractals, vol. 29, no. 4, pp. 942-947, 2006.

[38] W. J. Wu, Algorithms and Computation, Springer, Berlin, Germany, 1994. 




Advances in

Operations Research

mansans

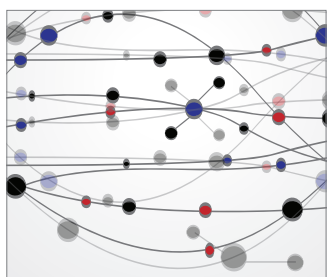

The Scientific World Journal

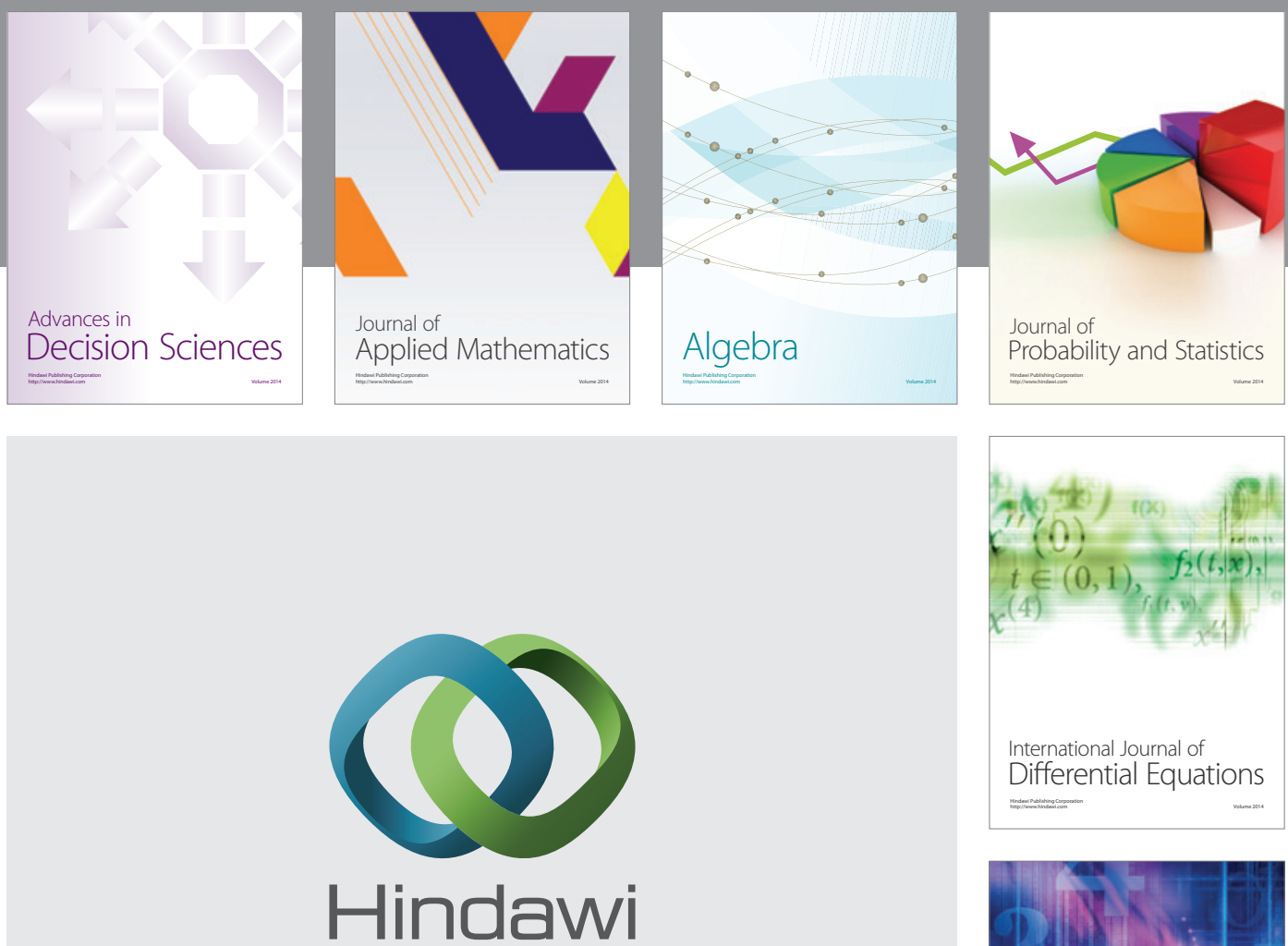

Submit your manuscripts at http://www.hindawi.com


Journal of

Function Spaces

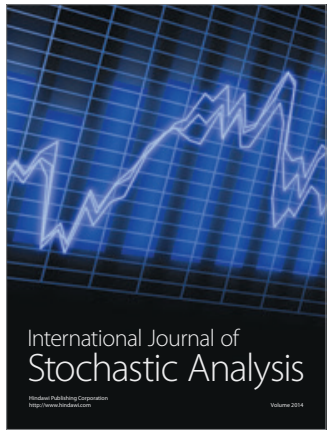


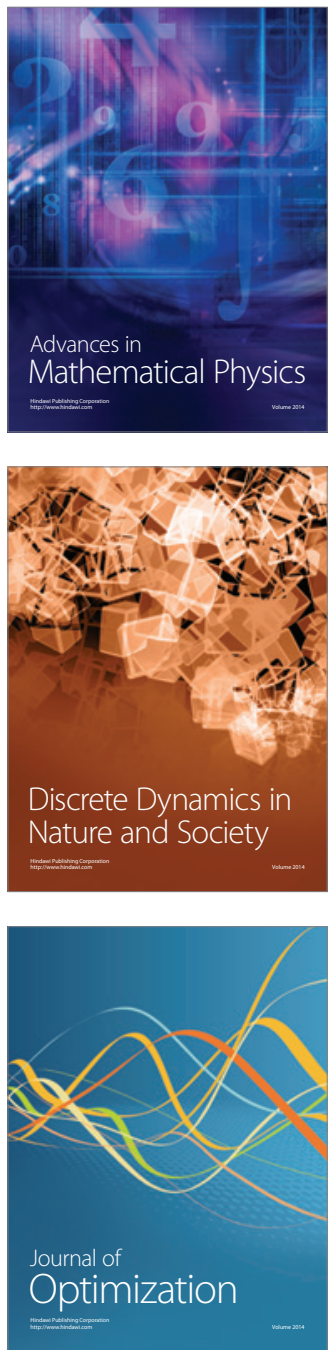